\title{
Design and Implementation of a Computerized User Authentication System for E-Learning
}

\author{
https://doi.org/10.3991/ijet.v15i09.12387 \\ Z. Faizal Khan $\left.{ }^{(}\right)$, Sultan Refa Alotaibi \\ Shaqra University, Shaqra, Kingdom of Saudi Arabia \\ faizalkhanesu.edu.sa
}

\begin{abstract}
The system based on formalized teaching with the help of electronic resources is called as E-learning. Using E-Learning in the university education systems has been growing rapidly based on the application of Information Technology (IT) designed for the substitute of traditional based learning approach. In terms of the E-learning based examinations, security constraints such as, identification of the user, misuse the passwords, unauthorized user instead of the authenticated one are available. Because of these disadvantages, the need of an authentication system is very important. The main challenge facing the security of E-learning environment is how to identify the unauthorized users from accessing and managing any platform. Therefore solving the problem of authenticating the valid user is very important. So, an intelligent examination system along with user authentication based on facial expression recognition is proposed in this paper. The proposed system can be classified in to three phases. The first phase is the design of facial database from the captured images. The second phase is the design of Artificial Intelligence (AI) based system for facial feature extraction and its classification using intelligent Agents. The final phase is the authentication of respective users by automatic identification of facial images. The results obtained shows that the proposed methodology has managed to validate the authenticated users and to provide an effective security in the E-learning platform.
\end{abstract}

Keywords - E-Learning, User Authentication, Intelligent Agents, Feature Extraction, Security.

\section{Introduction}

Information technology has become an important role the field of education. Recently, the E-Learning techniques have rapidly developed and there are many types of E-Learning based methodologies [1-4, 20]. Learning Management methods [7, 912] such as the traditional based, online based, and the workplace etc. [3-6] as well for education, through which everyone can perform the examinations. E-learning based on IT has become the necessary resource from which an instructor needs to handle the education because the instructor is the one who can develop the students by improving the skill of searching resources, then to apply them to their day to day activities. ELearning is a part of the learning activities [16, 17]. It is the easiest method of 
accessing the teaching materials especially for the students. It is also a method for connecting the instructors as well as the students. The instructor/lecturer can conduct online based examinations; he/she can evaluate and revert back the students with their marks.

E-Learning platform had some disadvantages such as the administrator or the system can't recognize the user who is accessing the system. Many E-Learning systems are running with the lack of such security issues. These Security requirements should be addressed by the developers so that, entire data and process of the Elearning will be safe from the threats and risks such as the intrusion and modification of data. These security requirements can also protect the hardware and software which are used in the E-Learning platform. Majority of the risks and threats can be avoided in the E-learning when it is being used by an authorized user. Due to this, the users and administrators of the E-Learning platform emphasizes on an effective user authentication and authorization system for it. Apart from this, the user authentication system also ensures the users identity and it will keep the records of all the users who are trying to access the system for further references.

In this paper, the intelligent agents proposed in this methodology identify the valid user by capturing the photos of the user, extracts the features and then compares it with the already extracted features which are present in the database. Based on the classification of features, the system will decide the user and will automatically allow the authorized user and also blocks the un-authorized users from accessing the system.

\subsection{Objectives of the proposed methodology}

Main objects of the proposed intelligent based examination system are as follows

- To have an insight into authenticating the users in E-learning platform

- To know various substructures which are used to examine the user authentication system based on intelligent agents. This includes studying its operational characteristics and the working environment.

- To design an intelligent based methodology for evaluating it.

- Modification of the current authentication system in E-Learning based on the proposed methodology.

This paper is organized as follows; section 2 depicts the literature review focusing on various methods proposed by earlier researchers, section 3 discussed the proposed methodology, its phases and the modules along with the system architecture, section 4 depicts the result and discussion section, conclusion and future works are shown in section 5 .

\section{Literature Review}

User Authentication is the process of verifying and authorizing an active user's identity. Security and user authentication are related in the E-Learning platform since unauthorized user can cause a potential threat to it. The main aim of user 
authentication is to ensure that the person who is interacting with the online examination system is a genuine user or not. Various researches have been done in the past $[16,19,20,21]$ to propose user authentication in the online examination system. Image processing is the process of analyzing and manipulating the digitized image, especially in the process of improving its quality. An image processing based user authentication framework is proposed in [11]. These framework aims to propose an authentication model E-learning that can authenticate the users through image processing based methods. This framework has several parts such as the creation of a dataset of faces for evaluating the proposed framework. Then, a histogram equalization process is used to enhancement the facial features. Third process is the feature descriptor selection using boosted cascade feature extraction methodology. Then, these extracted features will be saved to database. Final step will be the comparison of extracted features with the features already stores in the database for an effective identification of user.

Authors in [14] used the palm print as input in along with the user name and password as user authentication. Their methodology was a new authentication scheme that incorporates username/password with palm-based biometrics. Their method was good for authentication during the login phase. Merging the knowledge based authentication which contains the username, password along with the biometrics based authentication can use as an excellent transparent authentication scheme. The main drawbacks in this methodology is it requires an additional hardware such as the palm print reader to take the images from palm print and a centralized server to store and process the obtained palm print images. A novel adaptive based user authentication methodology in online examinations was developed in [7]. Their methodology improved the quality of already proposed examination system in [14] by creating a pattern for examination. A pre-assessment of the questions is done so that, the examination can be moderate as well as hard for the students. Answers are also prepared based on the examination pattern. A user authentication stagy is also improved in their methodology. In their methodology, the user authentication part is not implemented.

Authors in [15] proposed a novel framework for providing authentication in the online based examinations using voice recognition. In their research, voice of the user is acquired through a microphone so that the user can register for online. This framework is proposed such a way that it can be used for continuous authentication of online users. This framework can be useful for the people who are blind or to the people who can't use a keyboard in order to interact with the system. The main disadvantage of their proposed framework is that, it is susceptible to impersonation threats since it needs high sensitive voice recognition hardware and a signal processing system. Multiple assessments with formulas are proposed by in [11] in order to create serial examinations and also for evaluating continuously. Their method is based on multiple criteria. Their online based examination system can be used in the bank system. It can be gradually changed dynamically for other examination systems for the students' learning purposes. Authors in [13] authentication scheme for the online examinations. Their system provides the security to improve on-line examination by utilizing technologies such as biometric authentication, internet- 
firewall, cryptography, network protocol and object oriented paradigms. Their methodology targets an online based exam for Basic computer in university courses with students at particular locations, at a fixed time and same questions for all examinees at the restricted physical location of the examinees. In this methodology, combination of camera and a finger print scanner is employed to identify the students. Main disadvantage of their method is when a student or a faculty tries to access the system from different IP's, and then the system does not allow them to access.

Various researches have been done in the past to propose an effective E-learning system. Different types of online based examinations are developed in the past along with the user authentication system. Majority of the methods didn't perform well either in terms of additional hardware requirement or in terms of accuracy and its complexity. The biometrics based user authentication is not widely used since they are considered as intrusive and a violation of privacy of personal. In biometric based systems, the biometric data has to be saved in a centralized database. Many biometrics users are reluctant to give out their biometric data because they are worried how their biometric data will be used. Therefore, an efficient effective and external hardware reduced methodology for providing the authentication to user who is trying to access the E-Learning system is a must.

\section{Proposed Methodology}

Biometrics is a new technology that is used to enhance security in its applications. It is also defined as the identification of a user based on physiological and behavioral characteristics [12] Physiological biometrics are based on the user's physical characteristics which includes Facial images, facial IR thermograms, Hand (fingerprints, hand geometry, palm prints, hand IR thermograms), Eye (iris and retina), Ear, Skin, Dental, and DNA etc. This paper has used the facial images as Physiological biometrics input. The proposed intelligent authentication system consists of three phases 1) Data acquisition and storage; 2) Facial feature extraction and classification; 3) Comparison of features with the facial database. First phase is the Data acquisition and storage. In this phase, facial images of all the users who are expected to use the proposed system have to be saved in a facial database server. Then the System also asks the user to activate the live camera so that it can scan and detect faces. Second phase is the Facial feature extraction and classification. In this stage, the intelligent agents are used to extract the features from the acquired facial image. This is done by an agent-based image analysis [10, 201] method using AI.

Finally, comparison of the extracted features with the feature database is done to identify the face present which is present online. Overall architecture of the proposed methodology is shown in Figure 1. Overall architecture of the proposed system is shown in Figure 1 which accepts the user request through an authentication system. The authentication system is directly connected with the users who are using this intelligent examination system. It accepts the user credentials such as the username and password from the respective users. Users such as the students, teacher and the administrator can access the system using the required credentials. 


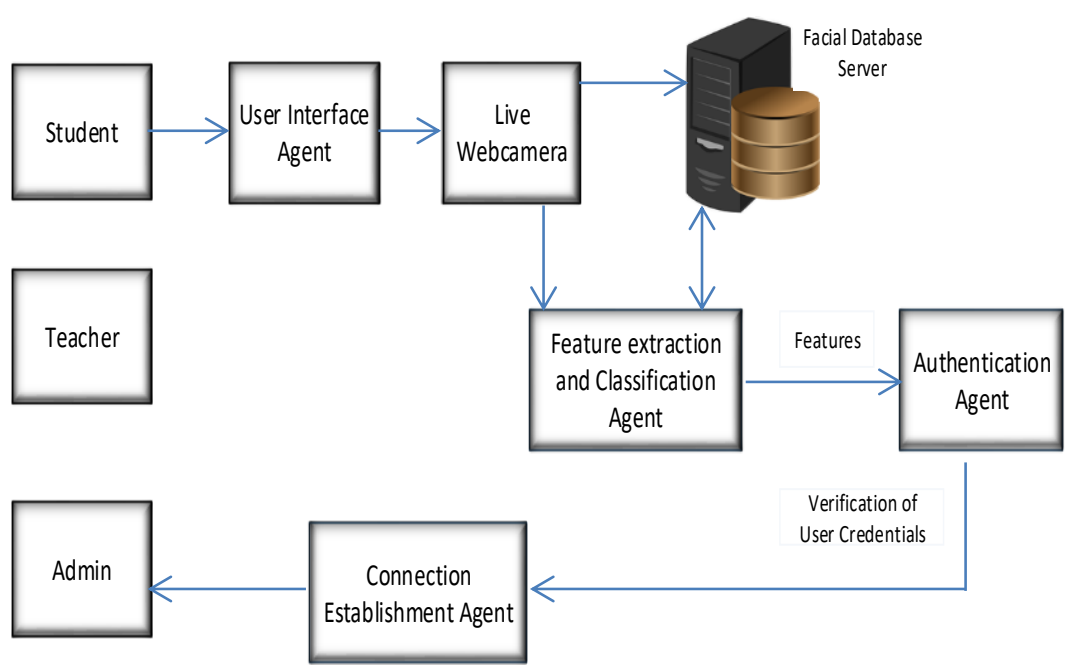

Fig. 1. Architecture of the proposed system

\subsection{Agent based systems:}

Agents perform actions based on the information they perceive from their environment [2], [17]. Agents gather information from their environment with the application of physical sensors or software sensors; they use this information to perform certain actions on their environment with the help of effectors or actuators as shown in figure 2 [17], [18], [19]. In this paper, agent-based image analysis is done by using the intelligent agents to determine the correct user. Various AI based agents such as the User interface agents, feature extraction and classification agent, authentication agents and the connection establishment agents were used to identify the faces of the user who is trying to access the system.

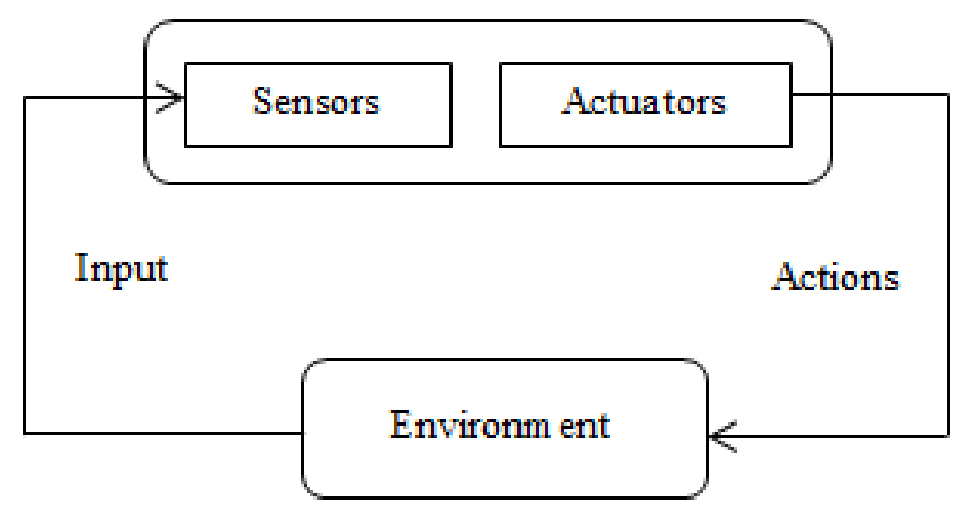

Fig. 2. Structure of an intelligent agent 


\subsection{User interface agent}

The user interface agent provides personalized assistance to users who are accessing the system. In this framework, main role of the user interface agent is to monitor the functions such as login, editing the profile and setting permission for the users for accessing the system. Login function monitors the users who are accessing the system. It manages the setting permission to different types of users such as the student, teacher or administrator.

\subsection{Data acquisition and storage}

In this phase, facial images of all the users who are expected to use the proposed system are saved in a facial database server. In order to increase the diversity of the data set, facial images with different deflection angles are included in the facial database. In the method, a facial database is used. The facial data set consists of images of faculties as well as the students along with their names and University registration number. The database consists of frontal face images of the user with deflected angles. Textural features of faces are already extracted for the images and are stored in the database for the process of training the feature extraction and classification agent. The feature extraction and classification agent extracts and classifies the features whether the features belongs to the authenticated user or not.

\subsection{Feature extraction and classification agent}

Many framework have been proposed in $[15,16,17]$ for intelligent agents. Feature extraction is a process that extracts a subset of original features. The feature extraction agents extracts the texture features from the captures input image. A typical feature extraction process consists of four basic steps namely, feature selecon, feature evaluation, stopping criterion, and features as the result. In this method, a well known classifier called as the Support Vector Machine (SVM) [22] was introduced as an automatic facial expression classifier. The support vector machineis an expression classifier which analyses the features as attributes in the data set. This classifier classifies the extracted features with the already stored features in the databse. Results of the classifier is wether features are matched or not. Output of the classifier is further passed towards the authentication agent.

Given a set of features $F=\{f 1, \ldots, f i, \ldots, f n\}$ the Feature extraction problem finds the texture features based on the following equation:

$$
\text { Energy }=\sum_{i j}(i, j)^{2}
$$

Algorithm for Feature Extraction

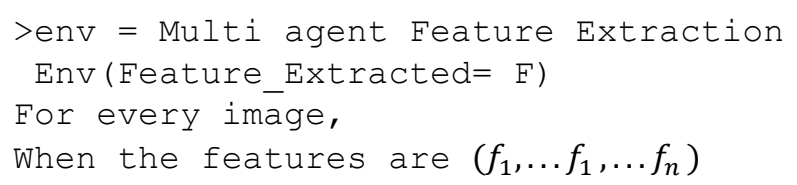




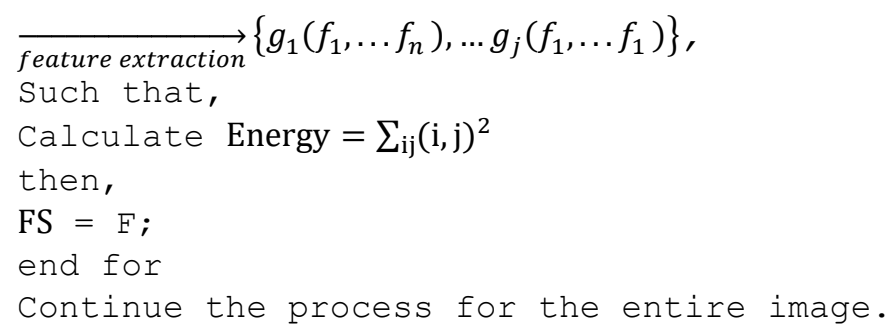

Algorithm for classification

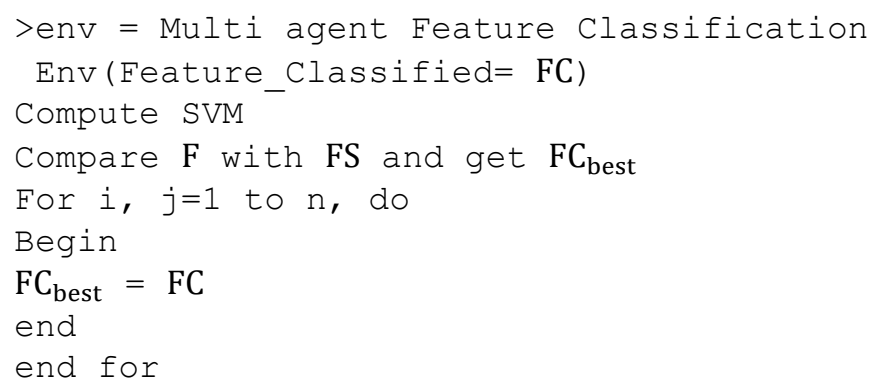

\subsection{5. Authentication Agent}

In this section, an AI based intelligent system is used for identify the faces based on the extracted facial features. The algorithm is trained with the features and its respective datasets present in the facial database server. Output of the classifier is given to the authentication agent. The authentication agent authenticates the user and grants permission to access it.

Algorithm for Authentication

Algorithm for authenticating the user and providing access to the system is as follows:

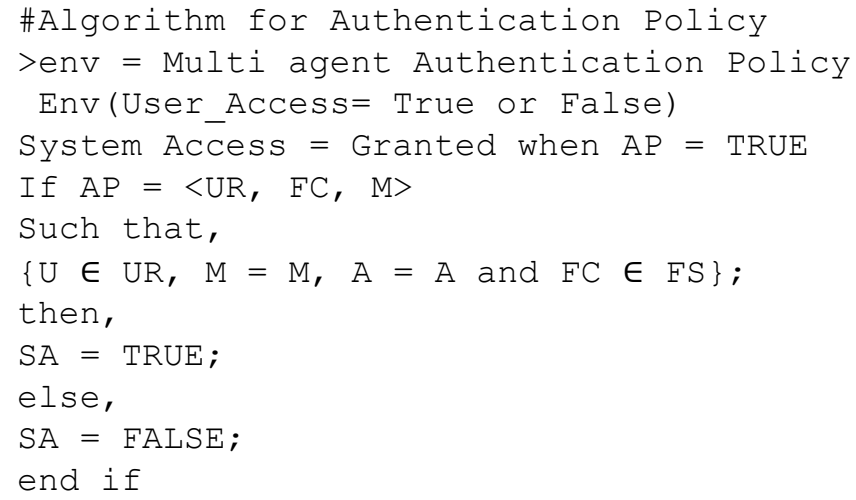

Algorithm for Accessing the System 


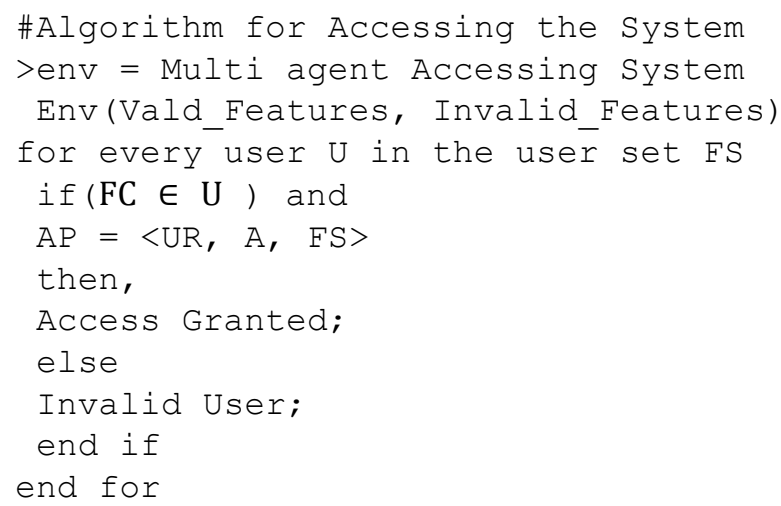

\subsection{Connection establishment agent}

When a user request a data, a connection is established between the user and the server side by the connection establishment agent. The credentials of requester such as the user-name/password which is present in the database is then extracted and it is verified from a list of valid user accounts present in it. The information about the established connection will be also stored in the database so that it can be to use for further references. The connection management agent is responsible for maintaining all the established connections which can be further used to retrieve previous data for the respective requests for further access.

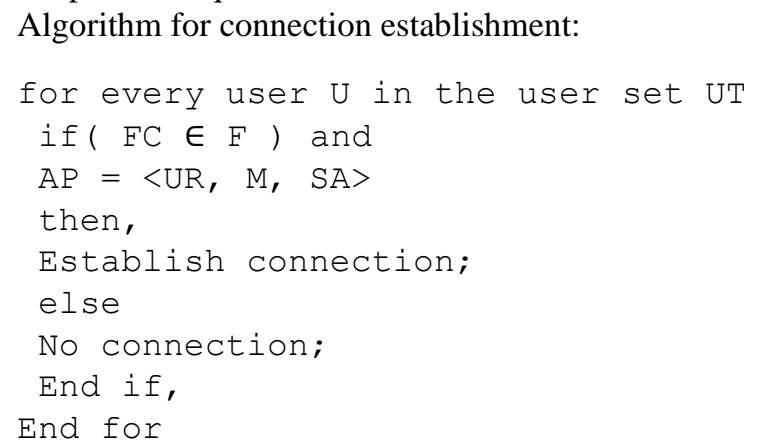

\subsection{Notations used}

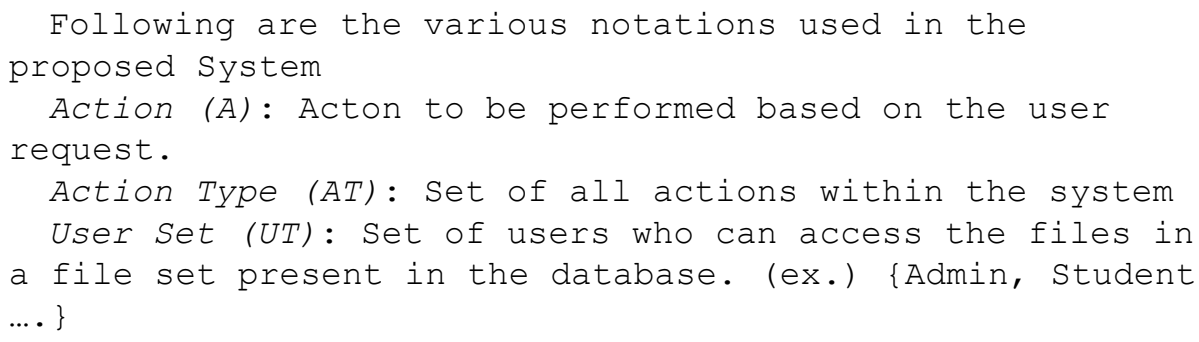




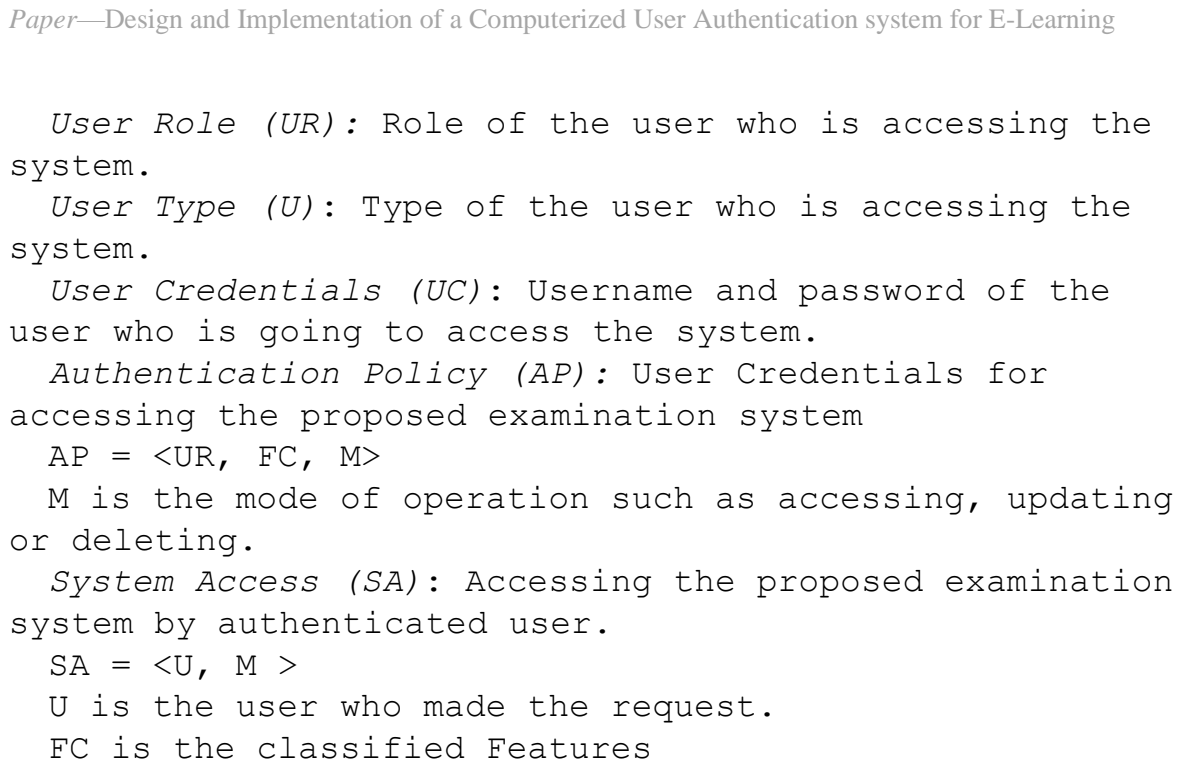

\section{$4 \quad$ Results and Discussion}

The proposed system requires a minimum hardware such as an i3 and above processor with $2 \mathrm{~GB}$ memory, 500GB hard disk. The operating system and software can be window 7 and other window operating system, an image input device: general anti-noise live camera. The proposed system is implemented in a way such that it can be used online with a live camera attached. Figures shown below are the various interfaces form the executed system. Figure 3 (a) shows the executing main interface of the proposed E-Learning system. It has two fields such as logging in and register. The users as student and administrator can register in the system. If the user is new, then he has to register as a new user. 


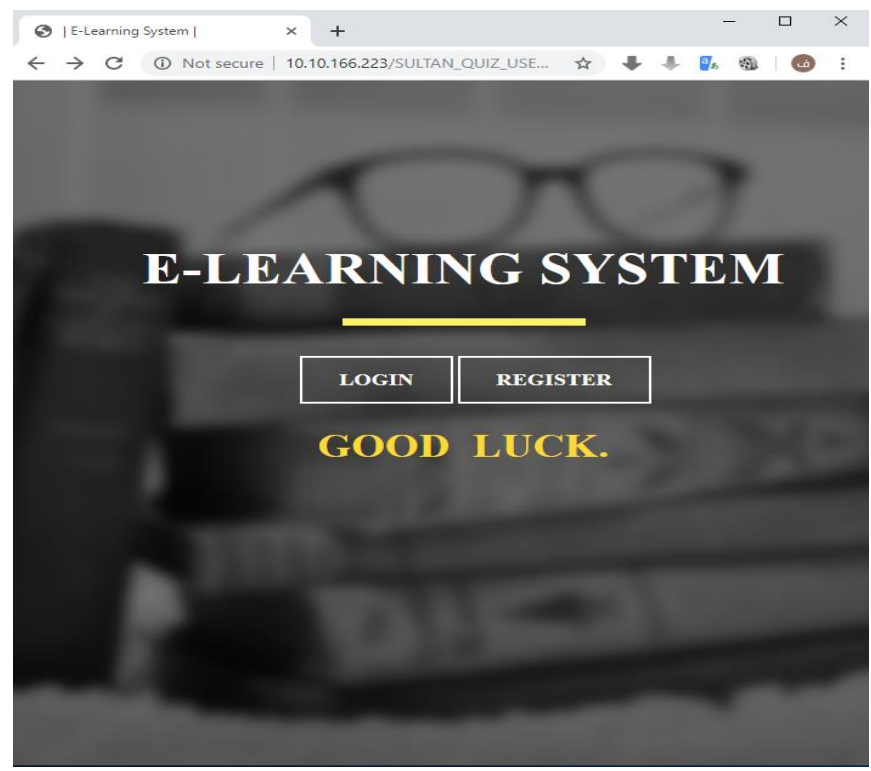

Fig. 3. (a) Main Interface of the proposed system

When the user clicks the register field, it opens a new interface for registration. It is shown in Figure 3 (b). All the necessary details such as the name, email id, desired password, University id etc. will be given along with the face input image. The Facial image given as input for registration is shown in Figure 3 (c).

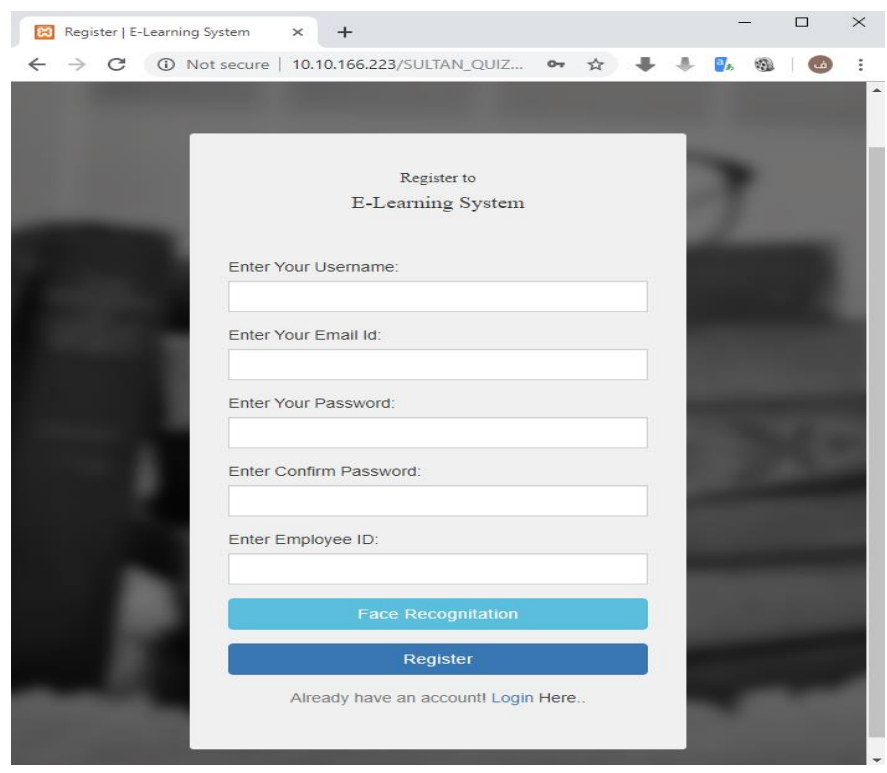

Fig. 3. (b) Interface of the user registration section 
When the user is new to the system, then he have to click the face registration field. The face registration field opens a new interface for capturing the face. In this interface, the facial image of user is taken as input to the system for user registration. Interface of the user registration section is shown in Figure 3 (d).

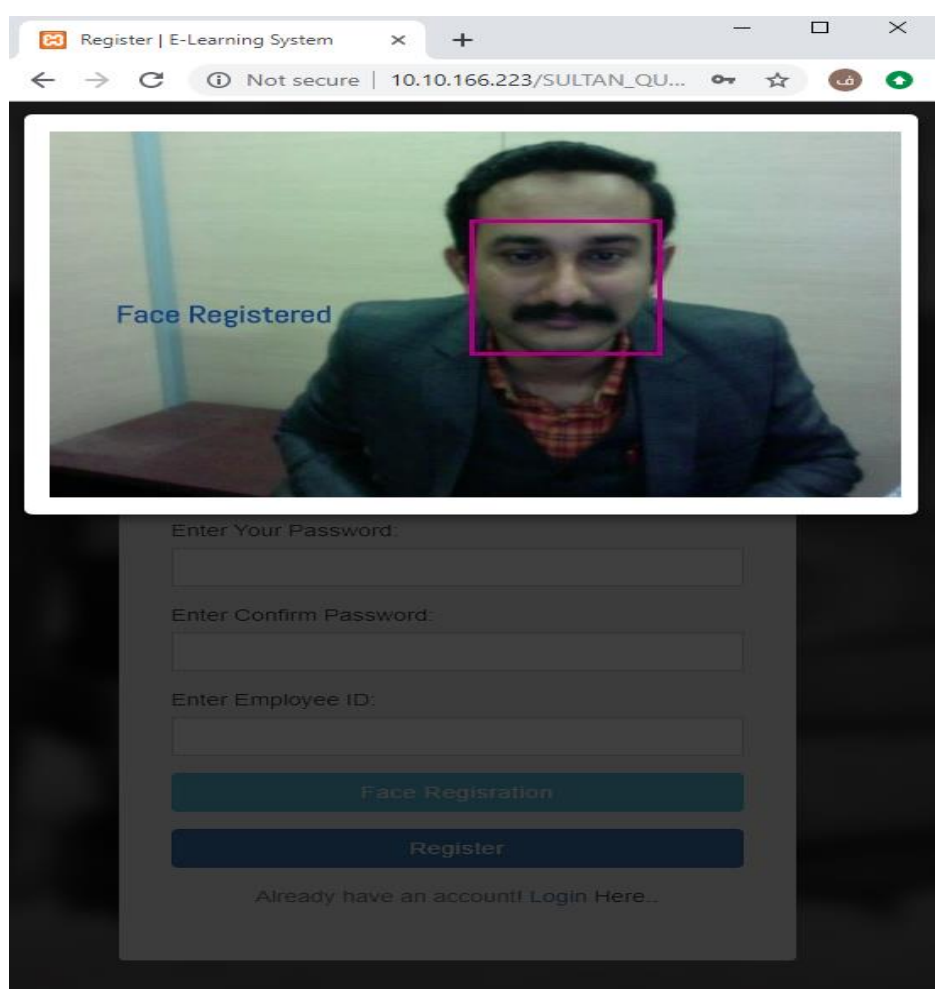

Fig. 3. (c) Interface of the user registration

When the user clicks the login field, it opens a new interface for logging in. In this interface, the facial image of user is processed as input to the system for user recognition. Interface of the user recognition section is shown in Figure 3 (d). 


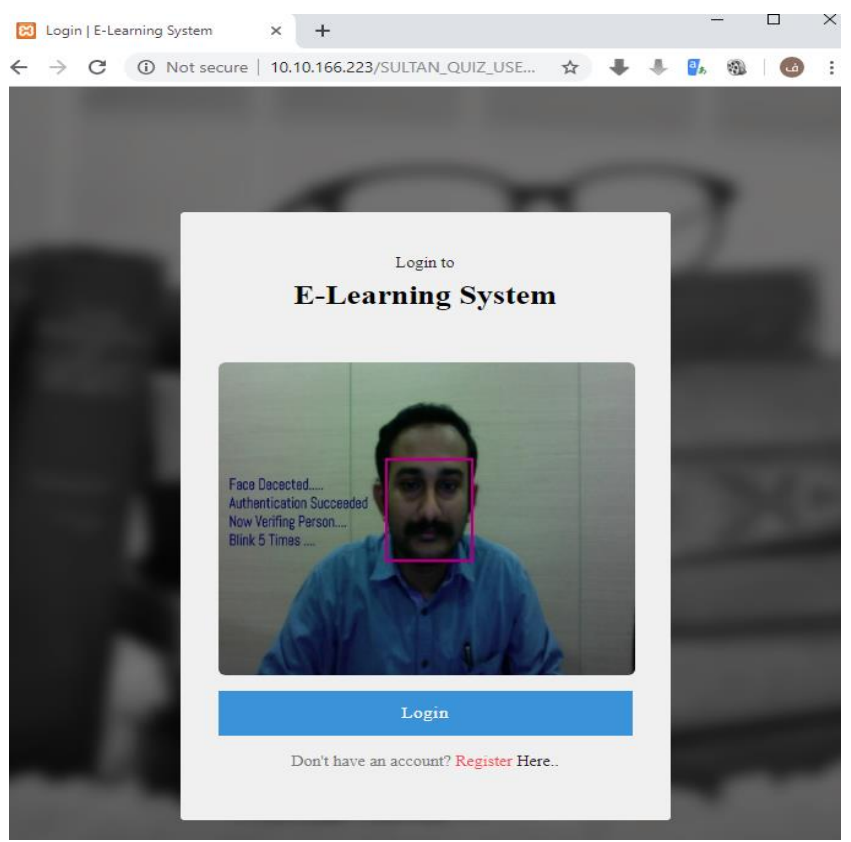

Fig. 3. (d) Interface of the user recognition at login

After recognizing the user, the system will automatically open the respective user section. The system will give access to the system based on the type of user. If it is a student, then the student page leads to the examination, marks and the feedback section. If the user is an administrator or a faculty, then the administrator page will be opened as shown in Figure 3 (e).

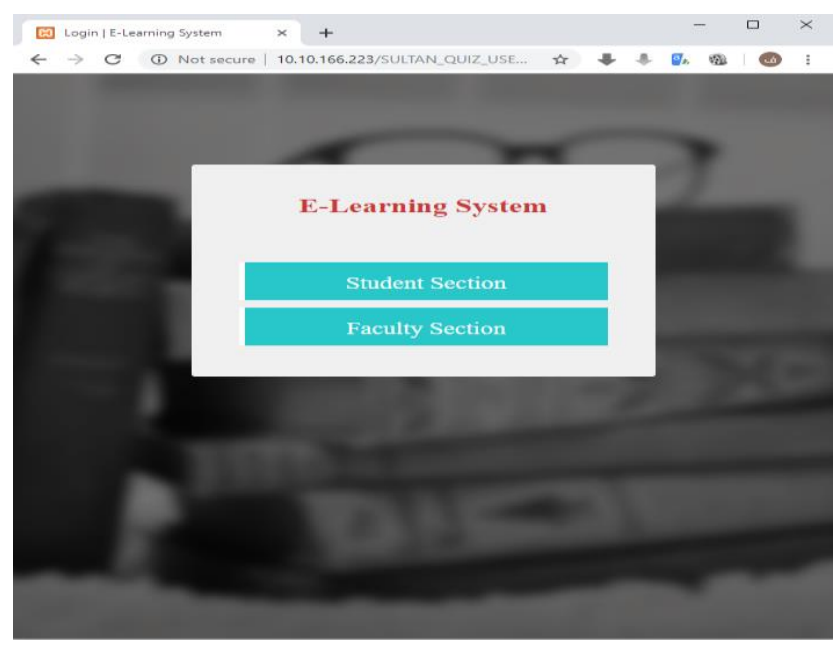

Fig. 3. (e) Interface of user after logging in 
The administrator section contains various fields such as insert courses, insert questions, modify the already available questions, result summary and the complete results section etc. will be displayed.

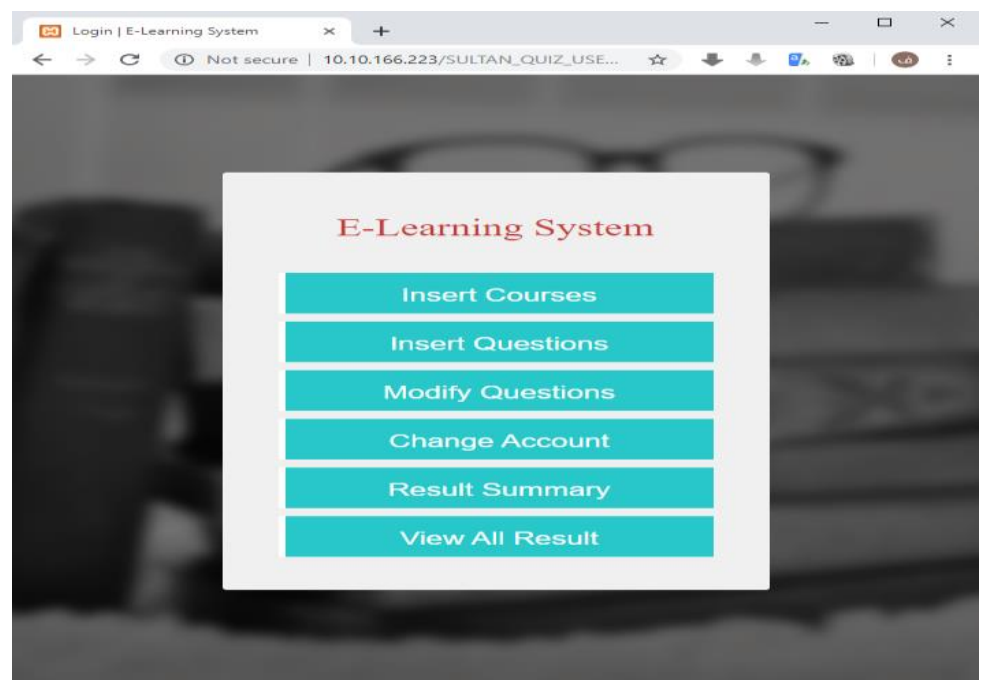

Fig. 3. (f) Interface for the faculty section

\section{Conclusion and Future Enhancement}

Application of E-Learning in the education system has been growing rapidly based on the application of IT designed for the substitute of traditional based learning approach. The online examinations conducted through this E-Learning platform should be secured especially from the unauthorized users trying to access the system for both the examination as well as administrating it. In order to make sure the access management, a novel authentication process using intelligent based agents is proposed in this paper. Various intelligent agents such as the user interface agent, feature extraction and classification agent, authentication agent and the connection establishment agent were used in this methodology. This model is novel since intelligent multi agents where used for the entire process of providing the access control. Intelligent agents present in this method perform different type of tasks. This agent based design aims at simple and efficient access control mechanism based on the user and their assigned roles. Future work can be the implementation of the proposed model with more datasets in order to calculate its efficiency and accuracy.

\section{References}

[1] Mushtaq Hussain, Wenhao Zhu, Wu Zhang, Syed Muhammad Raza Abidi, Student Engagement Predictions in an e-Learning System and Their Impact on Student Course 
Assessment Scores, Computational Intelligence and Neuroscience Volume 2018, Article ID 6347186. https://doi.org/10.1155/2018/6347186

[2] Nasim Abdulwahab Matar, Defining E-Learning Level of Use in Jordanian Universities Using CBAM Framework, International Journal of Emerging. Technology in. Learning, Vol 12, No 3, pp. 142-153, 2017. https://doi.org/10.3991/ijet.v12i03.6497

[3] S. Banerjee, N. J. Rao, and C. Ramanathan, "Designing item banks in alignment with course outcomes for engineering courses," in Proc. IEEE 8th International Conference on Technology for Education, 2016. https://doi.org/10.1109/t4e.2016.039

[4] Quadri Noorulhasan Naveed, Naim Ahmad, Critical Success Factors (CSFs) for CloudBased e-Learning, International Journal of Emerging. Technology in. Learning, Vol 14, No 1, pp. 140-149, 2019. https://doi.org/10.3991/ijet.v14i01.9170

[5] K. Hammond, Practical Artificial Intelligence for Dummies, 1st ed. Narrative Science, 2015, pp. 1-30.

[6] I.Portugal, P. Alencar, D. Cowan. (2015). The Use of Machine Learning Algorithms in Recommender Systems: A Systematic Review. [Online]. Available: https://arxiv.org/ftp/ arxiv/papers/1511/1511.05263.pdf

[7] K. Rajamani and V. Kathiravan, "An adaptive assessment system to compose serial test sheets using item response theory," in Proc. the 2013 International Conference on Pattern Recognition, Informatics and Mobile Engineering, 2013, pp. 120-124. https://doi.org/10. $\underline{1109 / i c p r i m e .2013 .6496458}$

[8] M. Wang, C. Wang, C. Lee, S. Lin, and P. Hung, "Type-2 Fuzzy set construction and application for adaptive student assessment system," in Proc. 2014 IEEE International Conference on Fuzzy Systems, 2014, pp. 888-894. https://doi.org/10.1109/fuzz-ieee.2014. $\underline{6891894}$

[9] E. Khater, A. Hegazy, and M. E. Shehab, "Ontology-based adaptive examination system in e-learning management systems," in Proc. 2015 IEEE 7th International Conference on Intelligent Computing and Information Systems, 2015, pp. 243-250. https://doi.org/10.11 09/intelcis.2015.7397228

[10] Peter Hofmann, Paul Lettmayer, Thomas Blaschke, Mariana Belgiu, Stefan Wegenkittl, Roland Graf, Thomas Josef Lampoltshammer, and Vera Andrejchenko, Towards a framework for agent-based image analysis of remote-sensing data, Int J Image Data Fusion. 2015 Apr 3; 6(2): 115-137. https://doi.org/10.1080/19479832.2015.1015459

[11] Sucianna Ghadati Rabiha, Hendro, Sasmoko, Noerlina and Hanry Ham, Image Processing Model Based E- Learning for Students Authentication, 2017 IEEE International Conference on Information Management and Technology, 15-17 November 2017, pp.187191. https://doi.org/10.1109/icimtech.2017.8273535

[12] Nader Abdel Karim, Zarina Shukur, Using Preferences as User Identification in the Online Examination, International journal on Advanced Science Engineering and Information technology, vol.6. No. 6, 2016. https://doi.org/10.18517/ijaseit.6.6.1412

[13] Mohammad A Sarrayrih, Mohammed Ilyas, Challenges of Online Exam, Performances and problems for Online University Exam, IJCSI International Journal of Computer Science Issues, Vol. 10, Issue 1, No 1, January 2013

[14] Al-Saleem, S.M. and H. Ullah, 2014. considerations and recommendations in computerbased testing. Sci. World J.

[15] Rudrapal, D., 5. Das, 5. Debbarma, N. Kar and N. Debbarma, 2012. Voice recognition and authentication as a proficient biometric tool and its application in online exam for $\mathrm{PH}$ people. Int. J. Comput. Appl., 39: 6-12. https://doi.org/10.5120/4870-7297

[16] T. Kawamata, T. Ishii, S. Fujimori, and T. Akakura, "Stu- dent authentication by updated facial information with weighting coefficient in e-Learning," IEEE Region 10 An- nual 
International Conference, Proceedings/TENCON, pp. 551-555, 2017. https://doi.org/10.11 09/tencon.2016.7848061

[17] Addi Ait-Mlouk, Fatima Gharnati, Tarik Agouti, Multi-agent-based modeling for extracting relevant association rules using a multi-criteria analysis approach, Vietnam Journal of Computer Science, Volume 3, Issue 4, pp 235-245, 2016. https://doi.org/10.10 07/s40595-016-0070-4

[18] Shaheen A. Abdulkareem, Yaseen T. Mustafa, Ellen-Wien Augustijn, Tatiana Filatova, Bayesian networks for spatial learning: a workflow on using limited survey data for intelligent learning in spatial agent-based models, GeoInformatica, , Volume 23, Issue 2, pp 243-268, 2019. https://doi.org/10.1007/s10707-019-00347-0

[19] Nacéra Benamrane, Samir Nassane, Medical Image Segmentation by a Multi-Agent System Approach, German Conference on Multiagent System Technologies-MATES 2007, pp 49-60. https://doi.org/10.1007/978-3-540-74949-3_5

[20] Noorulhasan Naveed Quadri, AbulHafeez Muhammed, Sumaya Sanober, Mohamed Rafik N. Qureshi, Asadullah Shah, Barriers Effecting Successful Implementation of E-Learning in Saudi Arabian Universities, International Journal of Emerging. Technology in. Learning, Vol 12, No 6, pp. 94-107, 2017. https://doi.org/10.3991/ijet.v12i06.7003

[21] A. M. Mogus, I. Djurdjevic, and N. Suvak, "The impact of student activity in a virtual learning environment on their final mark," Active Learning in Higher Education, vol. 13, no. 3, pp. 177-189, 2012. https://doi.org/10.1177/1469787412452985

[22] Yanhui Guo, Xijie Yin Xuechen Zhao, Dongxin Yang, Yu Bai, Hyperspectral image classification with SVM and guided filter, EURASIP Journal on Wireless Communications and Networking, December 2019. https://doi.org/10.1186/s13638-019$\underline{1346-\mathrm{Z}}$

\section{Authors}

Dr. Z. Faizal is currently working as an assistant professor at the Department of Computer Science in the College of Computing and Information Technology (CCIT), Shaqra University, the Kingdom of Saudi Arabia. His research interests includes Artificial Intelligence, Intelligent based Systems, Image Processing and pattern Recognition. Currently he is working in a project financed under Shaqra University, grant Nr. D180000/G01/N055.

Dr. Sultan Refa Alotaibi, is an Assistant Professor and Dean in the College of Computing and Information Technology (CCIT) at Shaqra University, Kingdom of Saudi Arabia. His research interests include E-Government, E-Services, E-Health and Knowledge Management. He continued to contribute also to the development of various projects and systems; much of his work has been in embracing digital transformation and improving the current technology to solve challenges facing the College of Computing and Information Technology.

Article submitted 2019-11-16. Resubmitted 2020-01-10. Final acceptance 2020-01-13. Final version published as submitted by the authors. 